\title{
Gravitational collapse from smooth initial data with vanishing radial pressure
}

\author{
Ashutosh Mahajan* Rituparno Goswami $\dagger$ and Pankaj S. Josh $\oplus$ \\ Tata Institute for Fundamental Research, \\ Mumbai, India
}

\begin{abstract}
We study here the spherical gravitational collapse assuming initial data to be necessarily smooth, as motivated by the requirements based on physical reasonableness. A tangential pressure model is constructed and analyzed in order to understand the final fate of collapse explicitly in terms of the density and pressure parameters at the initial epoch from which the collapse develops. It is seen that both black holes and naked singularities are produced as collapse end states even when the initial data is smooth. We show that the outcome is decided entirely in terms of the initial data, as given by density, pressure and velocity profiles at the initial epoch, from which the collapse evolves.
\end{abstract}

PACS numbers: 04.20.Dw, 04.70.-s, 04.70.Bw

\section{INTRODUCTION}

An important question in black hole physics is whether it would be possible to avoid the naked singularities forming as gravitational collapse end states by imposing various possible physically reasonable conditions on the collapsing configurations. When a sufficiently massive star exhausts its nuclear fuel, it must collapse endlessly. From such a perspective gravitational collapse has been studied within the framework of general relativity extensively for various forms of matter such as dust, radiation collapse, perfect fluids, massless scalar fields (see e.g. [1] and references therein), to find that both black holes (BH) and naked singularities (NS) result as collapse end states.

Such collapse studies do assume physically reasonable conditions such as an energy condition, the regularity of initial data, and such others. It is however, not ruled out that further conditions, mainly motivated by physical considerations, might possibly help ruling out naked singularities, and if this turns out to be the case, this is certainly worth exploring in view of the basic importance of this question in the theory and applications of black hole physics.

Our purpose here is to examine if both $\mathrm{BH} / \mathrm{NS}$ phases of collapse end states would still occur, when in order to make the model physically more relevant, we consider only smooth initial profiles and also include non-zero pressures for the collapsing cloud. That is, the initial density and the radial and tangential pressures are assumed to be smooth, $C^{\infty}$ functions of the radial co-ordinate $r$. While the Einstein equations by themselves do not impose such conditions on the collapse (where the metric and other functions are basically required to be $C^{2}$ differentiable only), smoothness of initial data is frequently considered to be desirable, especially in view of the numerical evolutions of collapse models related to scalar field collapse [2]. Also, when the density and pressures are smooth functions, there will be, for example, no 'cusps' present initially at the center of the collapsing cloud. Again, while we do not know whether the central cores of high density collapsing clouds admit such features or not, it may be a good assumption to make on physical grounds, and then to examine the consequences in terms of the collapse end states.

Our second purpose here is to examine explicitly, how the collapse outcomes are affected when a non-vanishing pressure is taken into account. For the case of vanishing pressures, the outcome of dust collapse has been known with or without assumptions such as smoothness of initial density distributions [3]. Here the initial values of density profile and the velocities of the collapsing shells fully determine the collapse end state in terms of either a black hole or a naked singularity (see e.g. [4]). The situation is much less explored when pressures are present. We examine below a collapse model with a non-vanishing but smooth tangential pressure present. It is then seen explicitly that in fact the smoothness of the initial profiles cannot restore the Cosmic Censorship Conjecture (CCC) which forbids occurrence of naked singularities in collapse, and that there exist non-zero measure of initial data sets which lead to outcomes in terms of either BH or NS. The interesting feature about the model we construct in Section III is that despite the presence of pressure, the final state is determined entirely in terms of the density and pressure gradients specified at the initial epoch itself, as in the case of dust collapse.

The outline of the paper is as follows. In Section II we discuss the collapse equations and the regularity conditions. In Section III, a tangential pressure model is constructed and it is demonstrated how the given sets of initial value

\footnotetext{
*Electronic address: ashutosh@tifr.res.in

${ }^{\dagger}$ Electronic address: goswami@tifr.res.in

‡Electronic address: psj@tifr.res.in
} 
parameters such as the initial density and tangential pressure values, decide the collapse final state. The behavior of the tangential pressure near singularity is discussed in Section IV, and the nature of singularity is examined in Section V. Some conclusions are outlined in Section VI.

\section{EINSTEIN EQUATIONS, REGULARITY AND ENERGY CONDITIONS}

We use the polar coordinates $(t, r, \theta, \phi)$ to write the spherically symmetric metric as,

$$
d s^{2}=-e^{2 \nu(t, r)} d t^{2}+e^{2 \psi(t, r)} d r^{2}+R^{2}(t, r) d \Omega^{2}
$$

where $d \Omega^{2}$ is the line element on a two-sphere. Also we take the energy-momentum tensor to be diagonal for the collapsing Type I matter field (that is, the frame is a comoving coordinate system) which is given by,

$$
T_{t}^{t}=-\rho ; T_{r}^{r}=p_{r} ; T_{\theta}^{\theta}=T_{\phi}^{\phi}=p_{\theta}
$$

This is a fairly general class of matter fields, which includes various known physical forms of matter 5]. The quantities $\rho, p_{r}$ and $p_{\theta}$ are the density, radial pressure and the tangential pressure respectively. We take the matter field to satisfy weak energy condition, that is, the energy density as measured by any local observer be non-negative, and so for any timelike vector $V^{i}$ we have,

$$
T_{i k} V^{i} V^{k} \geq 0
$$

This amounts to,

$$
\rho \geq 0 ; \rho+p_{r} \geq 0 ; \rho+p_{\theta} \geq 0
$$

The dynamical evolution of the system is determined by the Einstein equations and for the metric (1) these are given as

$$
\begin{gathered}
\rho=\frac{F^{\prime}}{R^{2} R^{\prime}}, \quad p_{r}=-\frac{\dot{F}}{R^{2} \dot{R}} \\
\nu^{\prime}\left(\rho+p_{r}\right)=2\left(p_{\theta}-p_{r}\right) \frac{R^{\prime}}{R}-p_{r}^{\prime} \\
-2 \dot{R}^{\prime}+R^{\prime} \frac{\dot{G}}{G}+\dot{R} \frac{H^{\prime}}{H}=0 \\
G-H=1-\frac{F}{R}
\end{gathered}
$$

where $\left({ }^{*}\right)$ and $\left({ }^{\prime}\right)$ represent partial derivative with respect to $t$ and $r$ respectively and,

$$
G(r, t)=e^{-2 \psi}\left(R^{\prime}\right)^{2}, \quad H(r, t)=e^{-2 \nu} \dot{R}^{2}
$$

Here $F(r, t)$ is an arbitrary function, and in spherically symmetric spacetimes, it is called the mass function of the collapsing cloud, which may be interpreted to give the total mass within the shell of comoving radius $r$. In order to preserve the regularity at the initial epoch, $F\left(t_{i}, 0\right)=0$, that is, the mass function should vanish at the center of the cloud. It can be seen from the equation (5) that the density of the matter blows up when $R=0$ or $R^{\prime}=0$. Here the case $R^{\prime}=0$ corresponds to the shell-crossing singularities. However, it is widely believed (see e.g. [6]) that these singularities can be possibly removed from the spacetime as they are typically gravitationally weak. Hence, we shall consider here only the shell-focusing singularities which occur at $R=0$, where the physical radius of all the matter shells goes to a zero value. Now let us use the scaling independence of the coordinate $r$ to write,

$$
R(t, r)=r v(t, r)
$$


and we have,

$$
v\left(t_{i}, r\right)=1 ; v\left(t_{s}(r), r\right)=0 ; \dot{v}<0
$$

where $t_{i}$ and $t_{s}$ stand for the initial and the singular epochs respectively. The condition $\dot{v}<0$ signifies that we are dealing with collapse situations only. This means we scale the radial coordinate $r$ in such a way that at the initial epoch $R=r$, and at the singularity, $R=0$. The advantage that the introduction of this new variable $v$ offers is that the regular center at $r=0$ (where we also have $R=0$ ) is now distinguished from the genuine singularity at $R=0$ in that, we now have $v=1$ at the initial epoch, and $v=0$ at the singular epoch $R=0$, but at all other epochs in-between $v$ has a non-zero finite value for all values of $r$.

From the point of view of dynamic evolution of initial data, at the initial epoch $t=t_{i}$, we now have five arbitrary functions of coordinate $r$ as given by,

$$
\begin{gathered}
\nu\left(t_{i}, r\right)=\nu_{0}(r) ; \quad \psi\left(t_{i}, r\right)=\psi_{0}(r) ; \quad \rho\left(t_{i}, r\right)=\rho_{0}(r) \\
p_{r}\left(t_{i}, r\right)=p_{r_{0}}(r) ; \quad p_{\theta}\left(t_{i}, r\right)=p_{\theta_{0}}(r)
\end{gathered}
$$

We note that all the initial data represented by the equation (12) are not mutually independent, as from equation (6) we get,

$$
\nu_{0}(r)=\int_{0}^{r}\left(\frac{2\left(p_{\theta_{0}}-p_{r_{0}}\right)}{r\left(\rho_{0}+p_{r_{0}}\right)}-\frac{p_{r_{0}}^{\prime}}{\rho_{0}+p_{r_{0}}}\right) d r
$$

Now, to preserve the regularity and smoothness of the initial data let us make some assumptions about the initial pressures at the regular center $r=0$. Let the gradients of pressures vanish at the center, that is, $p_{r_{0}}^{\prime}(0)=p_{\theta_{0}}^{\prime}(0)=0$. The difference between radial and tangential pressures at the center should also vanish, i.e. $p_{r_{0}}(0)-p_{\theta_{0}}(0)=0$. With these physical assumptions, and from equation (13), it is evident that $\nu_{0}(r)$ has the form,

$$
\nu_{0}(r)=r^{2} g(r)
$$

where $g(r)$ is at least a $C^{1}$ function of $r$ at $r=0$, and at least a $C^{2}$ function for $r>0$. Hence we see that we have a total of five field equations with seven unknowns, $\rho, p_{r}, p_{\theta}, \psi, \nu, R$, and $F$, giving us the freedom of choice of two free functions. Selection of these functions, subject to the given initial data and weak energy condition, determines the matter distribution and metric of the space-time and thus leads to a particular dynamical collapse evolution of the initial data. For collapsing models we have $\dot{R}<0$.

\section{A COLLAPSE MODEL WITH VANISHING RADIAL PRESSURE AND NON-VANISHING TANGENTIAL PRESSURE}

The spherically symmetric collapse models, where the radial pressure is taken to be vanishing, but the tangential pressure could be non-zero have been studied in some detail over past years [7]. The main motivation here was, if pressures are introduced within a collapsing cloud, one would like to understand how the situation differed from the dust collapse, especially in terms of formation of black holes and naked singularities as collapse end states. The Einstein cluster [8] is an example of such a cloud where tangential stresses are present. This is a spherically symmetric cluster of rotating particles where the motion of the particles is sustained by an angular momentum which has an average effect of creating a non-zero tangential stress within the cloud.

What is clear now as we study the models with non-vanishing tangential pressures is that both $\mathrm{BH} / \mathrm{NS}$ phases do develop as collapse end states, that is, the naked singularities of dust collapse do not go away with the introduction of tangential pressure. In that sense, these are stable to introduction of pressure. While these are existence results, we would like to understand how the nature of the final state will depend on the nature of the initial data given (see also [9] for the case of Einstein cluster).

In the following, we construct an explicit example of a collapse model with a non-vanishing tangential pressure, and it is shown that the nature of the singularity in this case can be determined explicitly in terms of the initial values of the density and pressure parameters, as given at the initial surface from which the collapse develops. This is then parallel to the dust case where such results are available.

As pointed out in the previous section, we choose the two allowed free functions, $F(t, r)$ and $\nu(t, r)$ in the following way,

$$
F(t, r)=r^{3} \mathcal{M}(r)
$$


that is, we choose the class of mass functions where $\mathcal{M}$ is a function of the radial coordinate $r$ only. Also we choose,

$$
\nu(t, r)=c(t)+\nu_{0}(R)
$$

Our purpose here is to examine to what extent the initial data determine collapse final fate, and hence we desire to construct here explicitly a specific model of collapse with non-vanishing tangential pressure in which case we will show that both BH and NS are possible as end states from smooth initial data, and that it is the initial data set which determines the final outcome. This is definitely a generalization over the dust models where initial profiles fully determine the collapse end state. Eq. (16), in spite of being a strong assumption, enables us in doing this.

Now putting equation (15) in equation (5), we get,

$$
\rho=\frac{3 \mathcal{M}+r\left[\mathcal{M}_{, r}\right]}{v^{2}\left(v+r v^{\prime}\right)} ; \quad p_{r}=0
$$

Thus we see that the given choice of the mass function ensures the radial pressure to vanish identically throughout the collapse. Also, it is evident that the density at the initial epoch is given by,

$$
\rho_{0}(r)=3 \mathcal{M}(r)+r \mathcal{M}(r)_{, r}
$$

It is clear that, in general, as $v \rightarrow 0, \rho \rightarrow \infty$. Thus the density blows up at the singularity $R=0$ which will be a curvature singularity as expected. Also using equation (16) in equation (7), we have,

$$
G(t, r)=b(r) e^{2 \nu_{0}(R)}
$$

Here $b(r)$ is another arbitrary function of $r$. In correspondence with the dust models, we can write,

$$
b(r)=1+r^{2} b_{0}(r)
$$

where $b_{0}(r)$ is the energy distribution function for the collapsing shells.

Now let us consider a smooth initial data, i.e. the initial density, pressure, and energy distributions are expressed as only even powers of $r$.

$$
\begin{gathered}
\rho\left(t_{i}, r\right)=\rho_{00}+\rho_{2} r^{2}+\rho_{4} r^{4}+\cdots \\
p_{\theta}\left(t_{i}, r\right)=p_{\theta_{2}} r^{2}+p_{\theta_{4}} r^{4}+\cdots \\
b_{0}(r)=b_{00}+b_{02} r^{2}+\cdots
\end{gathered}
$$

With the above form of smooth initial data to evolve the collapse, we can integrate the equation (13) and get,

$$
\nu_{0}(R)=p_{\theta_{2}} R^{2}+\frac{\left(p_{\theta_{4}}-\rho_{2} p_{\theta_{2}}\right)}{2} R^{4}+\cdots
$$

Using now equation (16) in equation (6), we get

$$
2 p_{\theta}=R \nu, R \rho
$$

Finally, using equations (15), (16) and (19) in equation (8), we have,

$$
\sqrt{R} \dot{R}=-a(t) e^{\nu_{0}(R)} \sqrt{\left(1+r^{2} b_{0}\right) R e^{2 \nu_{0}}-R+r^{3} \mathcal{M}}
$$

Here $a(t)$ is a function of time. By a suitable scaling of the time coordinate, we can always make $a(t)=1$. The negative sign is due to the fact that $\dot{R}<0$, which is the collapsing cloud condition.

Let us define a function $h(R)$ as,

$$
h(R)=\frac{e^{2 \nu_{0}(R)}-1}{R^{2}}=2 g(R)+\mathcal{O}\left(R^{2}\right)
$$


Using equation (27) in equation (26), we have after simplification,

$$
\sqrt{v} \dot{v}=-\sqrt{e^{4 \nu_{0}} v b_{0}+e^{2 \nu_{0}}\left(v^{3} h(r v)+\mathcal{M}\right)}
$$

Integrating the above equation, we get,

$$
t(v, r)=\int_{v}^{1} \frac{\sqrt{v} d v}{\sqrt{e^{4 \nu_{0}} v b_{0}+e^{2 \nu_{0}}\left(v^{3} h(r v)+\mathcal{M}\right)}}
$$

The time of singularity for a shell at a comoving coordinate radius $r$ is the time when the physical radius $R(r, t)$ becomes zero. The shells collapse consecutively, that is one after the other to the center as there are no shell-crossings. Taylor expanding the above function around $r=0$, we get,

$$
t(v, r)=t(v, 0)+\left.r \frac{d t(v, r)}{d r}\right|_{r=0}+\left.\frac{r^{2}}{2 !} \frac{d^{2} t(v, r)}{d^{2} r^{2}}\right|_{r=0}
$$

Let us denote,

$$
\mathcal{X}_{n}(v)=\left.\frac{d^{n} t(v, r)}{d r^{n}}\right|_{r=0}
$$

As we have taken the initial data with only even powers of $r$, the first derivatives of the functions appearing in above equations vanish at $r=0$, hence we have,

$$
\mathcal{X}_{1}(v)=0
$$

Now we can express the next coefficient $\mathcal{X}_{2}$ as,

$$
\mathcal{X}_{2}(v)=-\int_{v}^{1} \frac{\sqrt{v} d v\left[\Delta v^{5}+\frac{2}{3} p_{\theta_{2}} v^{2}+2 b_{02} v+\frac{\rho_{2}}{5}\right]}{\left(h v^{3}+b_{00} v+M\right)^{\frac{3}{2}}}
$$

where,

$$
\Delta=6 p_{\theta_{2}}^{2}+8 b_{02} p_{\theta_{2}}-\rho_{2} p_{\theta_{2}}
$$

When $\nu=0$, it can be seen from equation (25) that the tangential pressure vanishes and the model becomes like dust. The time $t(v, r)$ and coefficient $\mathcal{X}_{2}$ for the dust case are given as

$$
\begin{gathered}
t^{d}(v, r)=\int_{v}^{1} \frac{\sqrt{v} d v}{\sqrt{v b_{0}+\left(v^{3} h(r v)+\mathcal{M}\right)}} \\
\mathcal{X}_{2}^{d}(v)=-\int_{v}^{1} \frac{\sqrt{v} d v\left[2 b_{02} v+\frac{\rho_{2}}{5}\right]}{\left(b_{00} v+\mathcal{M}\right)^{\frac{3}{2}}}
\end{gathered}
$$

The important point to note here is that any given initial profile given in terms of the density and pressure values at the initial epoch completely determines the function $\mathcal{X}_{2}(v)$.

In general, for any constant $v$ surface we have,

$$
\sqrt{v} v^{\prime}=-\sqrt{v} \dot{v} \frac{d t}{d r}
$$

We see that the time taken for the central shell to reach the singularity is given as

$$
t_{s_{0}}=\int_{0}^{1} \frac{\sqrt{v} d v}{\sqrt{v b_{0}+v^{3} h(0)+\mathcal{M}_{0}}}
$$

From the above equation it is clear that for $t_{s_{0}}$ to be defined,

$$
b_{00} v+h(0) v^{3}+\mathcal{M}_{0}>0
$$

Hence the time taken for other shells to reach the singularity can be given by the expression,

$$
t_{s}(r)=t_{s_{0}}+\frac{1}{2} r^{2} \mathcal{X}_{2}(0)+\mathcal{O}\left(r^{3}\right)
$$




\section{BEHAVIOR OF TANGENTIAL PRESSURE NEAR THE SINGULARITY}

From the equations (24) and (25), it is clear that near the center the tangential pressure behaves as,

$$
p_{\theta} \sim \frac{r^{2}}{R^{\prime}}
$$

If we wish to calculate the limit of $p_{\theta}$ along any curve approaching the central singularity then in $(v, r)$ plane along all these curve $v \rightarrow 0$ as $r \rightarrow 0$. Then in $(v, r)$ plane the equation of all these radial curves near the center should have the form,

$$
v=k_{1} r^{\alpha} \quad(\alpha>0)
$$

Rewriting the equation of these curves in $(t, r)$ plane we have,

$$
\frac{d t}{d r}=\frac{\partial t}{\partial r}+\frac{\partial t}{\partial v}\left(\frac{d t}{d r}\right)_{\text {along the curve }}
$$

Now using (30) and calculating the limits on the given curve we finally get in $(t, r)$ plane that the above ingoing curve can be written as,

$$
t_{s_{0}}-t=r^{2} \mathcal{X}_{2}(0)+k_{2} r^{(3 \alpha / 2)}
$$

Now if $\mathcal{X}_{2}(0) \neq 0$ it can be easily shown that the limit of $p_{\theta}$ in (41) does not blow up, and the system behaves like dust essentially near the central singularity. In case of $\mathcal{X}_{2}(0)=0$ it can be shown that for the values of $\alpha>2$ the pressure blows up. However from the metric it can be calculated that if such curves are to be timelike then strictly $\alpha<2$. Hence we see that there exist no timelike radial curve along which the tangential pressure blows up at the central singularity. However there are a large class of spacelike radial directions, described by $\mathcal{X}_{2}(0)=0$ and $\alpha>2$, along which it blows up at the central singularity.

Alternatively, we can also look at the solution close to the center. In the limit $R \rightarrow 0$ (for the zero initial velocity profiles), the equation equation (26) takes the form,

$$
R \dot{R}^{2}=2 p_{\theta_{2}} r^{3} \mathcal{M}(r) R^{2}+r^{3} \mathcal{M}(r)
$$

which can be integrated as

$$
\frac{1}{\sqrt{c_{2}}} R^{\frac{3}{2}} F\left[\frac{1}{2}, \frac{3}{4}, \frac{7}{4},-\frac{c_{1}}{c_{2}} R^{2}\right]=t-t_{s}(r)
$$

where $F$ is hypergeometric function, $c_{1}=\frac{9}{2} p_{\theta_{2}} r^{3} M(r)$ and $c_{2}=\frac{9}{4} r^{3} M(r)$.

Expanding the hypergeometric function $F$ and keeping only the leading order term, we get the solution for $R$ as

$$
R=\left(\frac{3}{2}\right)^{\frac{2}{3}} r M(r)^{\frac{1}{3}}\left(t-t_{s}(r)\right)^{\frac{2}{3}}
$$

In the equation (25), if we expand $\nu$ and $\rho$ we see that near the central singularity in the limit $R \rightarrow 0$ the pressure $p_{\theta}$ goes as $\left(p_{\theta 2} \rho_{00}\right)\left(r^{2} / R^{\prime}\right)$. Now, in $(R, r)$ plane, along a curve $R=k r^{b}$ the quantity $R^{\prime}$ can be calculated from the above equation, and when we approach the central singularity we see that the tangential pressure blows up for $b>3$.

We see that close to the center, this curve can be timelike only if $b<3$. Thus for the curve $R=a r^{b}, p_{\theta}$ blows up only along spacelike directions. A similar analysis was carried out for the curves like $t-t_{\text {so }}=\alpha r^{\beta}$ and it was found that $p_{\theta}$ blows up for the value of $\beta$ for which the curve is spacelike. 


\section{NATURE OF THE SINGULARITY}

We need to determine now when there will be families of future directed outgoing null geodesics coming out of the singularity and when there will be none. In the case when such families do exist which terminate in the past at the singularity, and which could reach outside observers, then the singularity will be visible. In the case otherwise it is hidden within the black hole. Another way to look at this is through the apparent horizon and formation of trapped surfaces in the spacetime. As the collapse evolves, if the trapped surfaces form well in advance to the formation of the singularity, then the same will be covered. On the other hand, if the trapped surface formation is sufficiently delayed during the collapse then the singularity may be naked. The apparent horizon within the collapsing cloud is given by the equation, $R / F=1$, which gives the boundary of the trapped surface region of the space-time. If the neighborhood of the center gets trapped earlier than the singularity, then it is covered, otherwise it is naked with non-spacelike future directed trajectories escaping from it.

In order to consider the possibility of existence of such families, and to examine the nature of the singularity occurring at $R=0, r=0$ in this model, let us consider the outgoing null geodesic equation which is given by,

$$
\frac{d t}{d r}=e^{\psi-\nu}
$$

We now use here a method which is similar to that given in [4]. The singularity curve is given by $v\left(t_{s}(r), r\right)=0$, which corresponds to $R\left(t_{s}(r), r\right)=0$. Therefore, if we have any future directed outgoing null geodesics terminating in the past at the singularity, we must have $R \rightarrow 0$ as $t \rightarrow t_{s}$ along the same. Now writing equation (48) in terms of variables $\left(u=r^{\alpha}, R\right)$, we have,

$$
\frac{d R}{d u}=\frac{1}{\alpha} r^{-(\alpha-1)} R^{\prime}\left[1+\frac{\dot{R}}{R^{\prime}} e^{\psi-\nu}\right]
$$

Now in order to get tangent to the null geodesic in the $(R, u)$ plane, we choose a particular value of $\alpha$ such that the geodesic equation is expressed only in terms of $\left(\frac{R}{u}\right)$. A specific value of alpha is to be chosen which enables us to calculate the proper limits at the central singularity. For example, for $\mathcal{X}_{1}(0) \neq 0$ case, we can choose $\alpha=\frac{5}{3}$ and using equation (8), (and considering that $\dot{R}<0$ ), we get,

$$
\frac{d R}{d u}=\frac{3}{5}\left(\frac{R}{u}+\frac{\sqrt{\mathcal{M}_{0}} \mathcal{X}_{1}(0)}{\sqrt{\frac{R}{u}}}\right)\left(\frac{1-\frac{F}{R}}{\sqrt{G}[\sqrt{G}+\sqrt{H}]}\right)
$$

In the tangential pressure collapse model discussed in the previous section we have $\mathcal{X}_{1}(0)=0$, and hence we choose $\alpha=\frac{7}{3}$ so that when in limit $r \rightarrow 0, t \rightarrow t_{s}$ we get the value of tangent to null geodesic in the $(R, u)$ plane,

$$
\frac{d R}{d u}=\frac{3}{7}\left(\frac{R}{u}+\frac{\sqrt{M_{0}} \mathcal{X}_{2}(0)}{\sqrt{\frac{R}{u}}}\right) \frac{\left(1-\frac{F}{R}\right)}{\sqrt{G}(\sqrt{G}+\sqrt{H})}
$$

Now note that for any point with $r>0$ on the singularity curve $t_{s}(r)$, we have $R \rightarrow 0$ whereas $F$ (interpreted as mass of the object within the comoving radius $r$ ) tends to a finite positive value once the energy conditions are satisfied. Under the situation, the term $F / R$ diverges in the above equation, and all such points on the singularity curve will be covered as there will be no outgoing null geodesics from such points.

Hence we need to examine the central singularity at $r=0, R=0$ to determine if it is visible or not. That is, we need to determine if there are any solutions existing to the outgoing null geodesics equation, which terminate in the past at the singularity and in future go to a faraway observer, and if so under what conditions these exist. Note that if any outgoing null geodesics terminate at the singularity in the past then along the same, in the limit as $r \rightarrow 0, t \rightarrow t_{s}$ we then have from equation (26) $\dot{R}=0$, therefore $H=0$ and $G=1$ in this limit as $F / R$ vanishes. Let now $x_{0}$ be the tangent to the null geodesics in $(R, u)$ plane, at the central singularity, then it is given by,

$$
x_{0}=\lim _{t \rightarrow t_{s}} \lim _{r \rightarrow 0} \frac{R}{u}=\left.\frac{d R}{d u}\right|_{t \rightarrow t_{s} ; r \rightarrow 0}
$$

Using equation (51), we get,

$$
x_{0}^{\frac{3}{2}}=\frac{7}{4} \sqrt{\mathcal{M}_{0}} \mathcal{X}_{2}(0)
$$


In the $(R, u)$ plane, the null geodesic equation will be,

$$
R=x_{0} u
$$

while in the $(t, r)$ plane, the null geodesic equation near the singularity will be,

$$
t-t_{s}(0)=x_{0} r^{\frac{7}{3}}
$$

It follows that if $\mathcal{X}_{2}(0)>0$, then that implies that $x_{0}>0$, and we then have radially outgoing null geodesics coming out from the singularity, making the central singularity to be a visible one. On the other hand, if $\mathcal{X}_{2}(0) \leq 0$, we will have a black hole solution.

We have, however, already seen in equation (33), that the value of $\mathcal{X}_{2}(0)$ entirely depends upon the initial density and velocity profiles. Therefore, given any density and tangential pressure profiles of the collapsing matter, we can always choose an energy profile so that the end state of the collapse would be either a naked singularity or a black hole and vice-versa.

These features come out clearly in the Figures 1-3, where it is examined how $\mathcal{X}_{2}$ depends upon the various initial data values chosen. Here the function $\mathcal{X}_{2}(0)$ is plotted with different values of initial density, tangential pressure and energy profiles. It is seen that there exist distinct regions in the initial data space where a certain region evolves to a naked singularity outcome, whereas the other part of the region evolves to a black hole. In the figures the red colored surface shows positive values of $\chi_{2}$. Whenever the initial data values lie in this region, null geodesics from the center come out of the central singularity, as a result creating the presence of a naked singularity as the collapse outcome. The green colored region represents black hole. The phase separation is clearly seen. It is to be noted that in case of a naked singularity, the singularity curve at the center, as given by equation (40), is an increasing function of $r$ as in that case $X_{2}(0)>0$, whereas a black hole solution gives a decreasing or constant curve for shells as the coordinate $r$ increases.

These plots also bring out another important feature arising in collapse, which is that the initial data sets which give rise to either a naked singularity or a black hole as final end state are open in the space of initial data from which the collapse evolves.

We note that even though the model considered here has non-zero pressure, this is subject to a strong assumption given by Eq. (16). This is equivalent to the ansatz $\nu(t, r)=\nu_{0}(R)$. A feature that the plots given here bring out is, in the space of initial data that is defined under these restrictions the $\mathrm{BH} / \mathrm{NS}$ outcomes are open sets. Though restricted as above, this scenario is more general as compared to dust collapse, in that pressure is included.

This of course does not mean that, in general, naked singularities would generically develop in any given collapse scenario. However, it is to be noted that the issues of genericity and stability are rather involved in Einstein's gravity, and are not so well-defined. Hence these must always be studied within the context of the limited models under consideration. It is to be hoped that such considerations will possibly throw some light on the nature of cosmic censorship statement which one may evolve eventually.

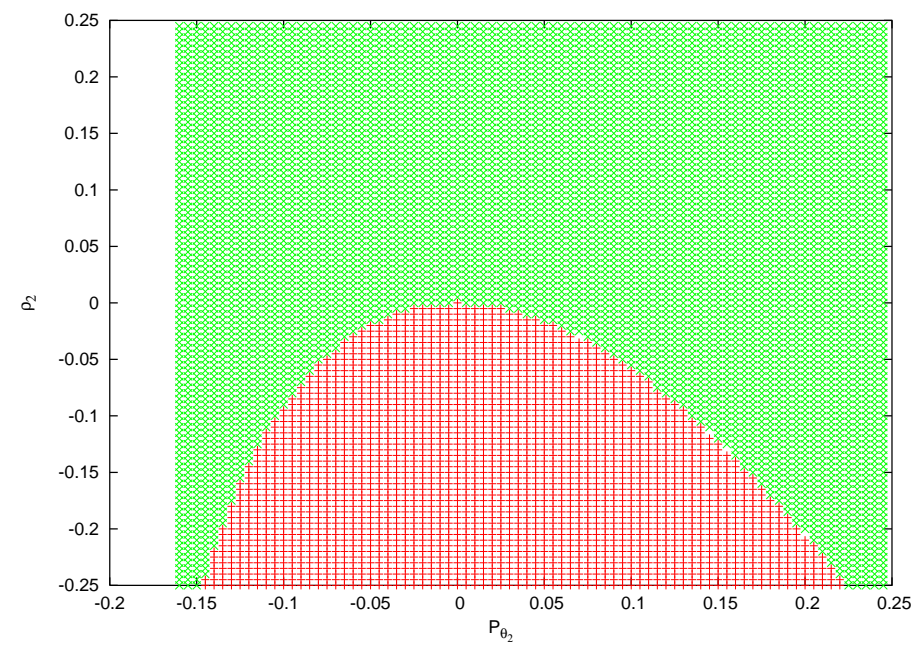

FIG. 1: Illustration of the dependence of end state of the collapse on initial density $\rho_{0}$ and tangential pressure $p_{\theta_{0}}$ for $b_{02}=0$. The red coloured region shows the initial data space which goes to NS, while the green region evolves to $\mathrm{BH}$. 


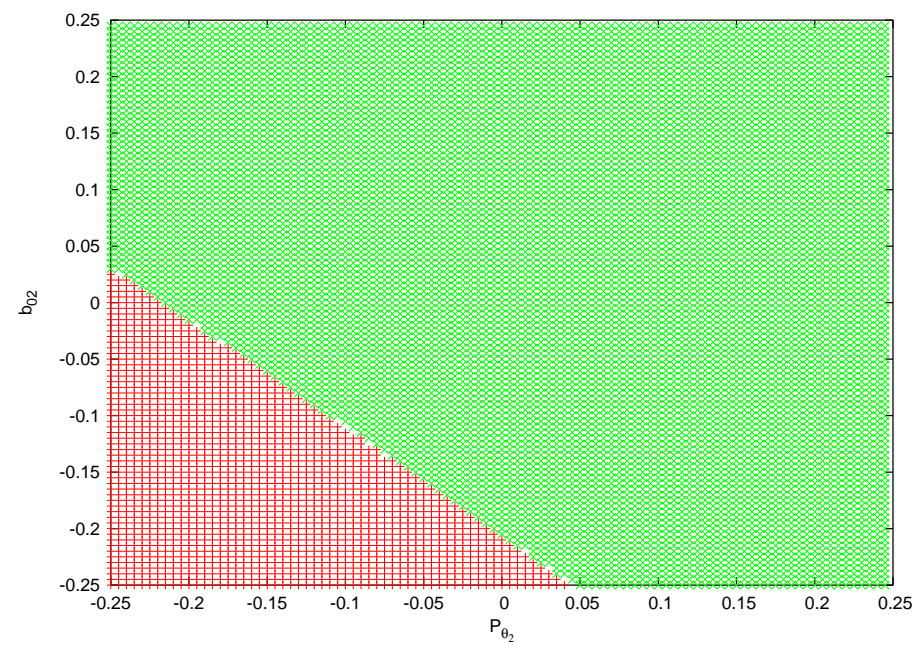

FIG. 2: Illustration of the dependence of end state of the collapse on initial tangential pressure $p_{\theta_{0}}$ and energy profile $b_{02}$ for $\rho_{2}=1$. The red coloured region shows the initial data space which goes to NS, while the green region evolves to BH.

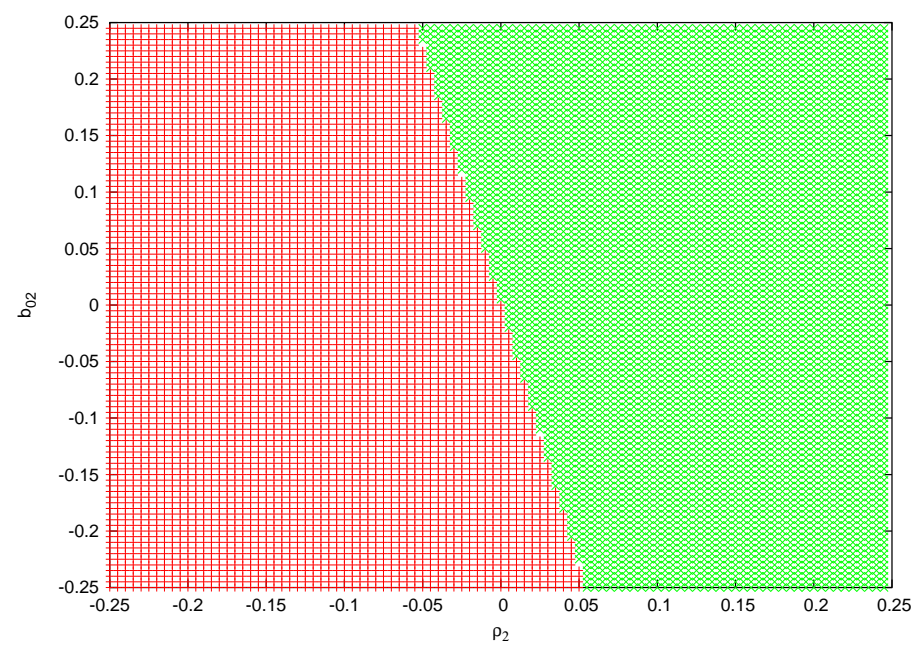

FIG. 3: Illustration of the dependence of end state of the collapse on initial density $\rho_{0}$ and energy profile $b_{02}$ for $p_{\theta_{2}}=0$. The red coloured region shows the initial data space which goes to NS, while the green region evolves to $\mathrm{BH}$.

\section{CONCLUSIONS}

We have considered here gravitational collapse from a smooth initial data. We have also included the presence of pressure while developing the collapse models. In this sense, the models here may be considered closer to being physically realistic, when compared to, for example, the dust collapse where the pressures are completely neglected. Some conclusions and remarks are summarized below.

1. We see that for the model considered here in Section III, where vanishing radial pressure is assumed and an additional assumption (16) is made, both $\mathrm{BH} / \mathrm{NS}$ phases develop as collapse end states. The important feature that we observe is the outcome is fully determined in terms of the initial profiles for density, pressures, and energy functions as given at the initial surface from which the collapse develops. This is completely due to the strong assumption (16). The point that is made here is, we have a construction or an example to show that naked singularities can still develop even when densities and pressures are restricted to be necessarily smooth. Secondly, as seen in the present case, the initial data alone could determine the final fate even when non-zero pressures are present in the collapsing cloud. It remains to be examined if this would hold in still wider classes of models other than those considered here.

2. We find in the above case, that there is an open initial data space which evolves to a naked singularity, and the 
same is true for a black hole end state as well. In this sense both these collapse outcomes are generic, within the framework of the models considered here.

3. The current analysis shows that only the requirements of smoothness of initial data, and the inclusion of pressures, are not sufficient by themselves to rule out naked singularities. As we see here, smooth initial data does give rise to NS.

4. Through out we have imposed the smoothness condition, however, it is worth noting that the data is required to be smooth only initially. The subsequent dynamical evolution of these initial matter profiles is then fully governed by the Einstein equations, and hence at later epochs during the collapse the density and pressure profiles may or may not continue to be smooth, and could very well develop non-analytic behaviour. It may be worth examining, especially when pressures are included, as to how the smoothness condition may be affected as the collapse evolves.

5. It is important to note that the analysis presented here only considered locally naked singularities. The null geodesics coming out from the singularity can in principle go to infinity, (i.e the singularity can be globally visible), or it could also happen that the trajectories could again fall back to the singularity. This in fact depends on the global behavior (i.e. for large values of $r$ ) of the functions concerned. In the case when a singularity is locally naked, one can always choose the global behavior of the rest of the dynamical functions in such a way that the singularity becomes globally visible.

[1] A. Krolak, Prog. Theor. Phys. Suppl. 136, 45 (1999). R. Penrose, in Black holes and relativistic stars, ed. R. M. Wald (University of Chicago Press, 1998); P. S. Joshi, Pramana 55, 529 (2000); M. Celerier and P. Szekeres, Phys.Rev.D65(2002) 123516; R. Giambo', F. Giannoni, G. Magli, P. Piccione, Commun.Math.Phys. 235 (2003) 545-563; T. Harada, H. Iguchi, and K. Nakao, Prog.Theor.Phys.107 (2002) 449-524.

[2] M. W. Choptuik, Phys. Rev. Lett.70, p9 (1993); A. M. Abrahams and C. R. Evans, Phys. Rev. Lett. 70, p.2980 (1993); C. R. Evans and J. S. Coleman, Phys. Rev. Lett.72, p.1782 (1994); M. D. Roberts, Gen. Relat. Grav.21, p.907 (1989); J. Traschen, Phys. Rev. D50, p.7144 (1994); P. R. Brady, Class. Quant. Grav. 11, p.1255; Phys. Rev. D51, p.4168 (1995); C. Gundlach, Phys. Rev. Lett. 75, p.3214 (1995); P. R. Brady, M. W. Choptuik, C. Gundlach, D. W. Neilsen, Class.Quant.Grav.19 (2002) 6359; E. Malec, Class. Quantum Grav.13, 1849 (1996).

[3] D. M. Eardley and L. Smarr, Phys. Rev. D 19, p.2239 (1979); D. Christodoulou, Commun. Math. Phys., 93, p.171 (1984); P. S. Joshi and I.H. Dwivedi, Phys. Rev. D47, p.5357 (1993).

[4] R. Goswami and P. S. Joshi, Phys. Rev. D69 (2004) 044002; R. Goswami and P. S. Joshi, Phys. Rev. D69 (2004) 104002.

[5] S. W. Hawking and G. F. R. Ellis, The large scale structure of spacetime, Cambridge Univ. Press, Cambridge (1973).

[6] C. J. S. Clarke, The analysis of spacetime singularities, Cambridge University Press, Cambridge (1993).

[7] G. Magli, Class. Quant. Grav. 14 (1997) 1937; Class. Quant. Grav. 15 (1998) 3215; S. M. C. V. Goncalves, S. Jhingan, G. Magli, Phys.Rev. D65 (2002) 064011; T.Harada, K.Nakao and H.Eguchi, Class.Quantum Grav. 16(1999) 2785-2796; R. Goswami and P. S. Joshi, Class. Quantum Grav. 19, 5229 (2002).

[8] A. Einstein, Ann. Math. 40, 4922 (1939); B. K. Datta, Gen. Relat. Grav. 1, 19 (1970); H. Bondi; Gen. Relat. Grav. 2, 321 (1971).

[9] T.Harada, H.Iguchi and K.Nakao, Phys.Rev.D58 (1998) 041502; S.Jhingan and G.Magli, Phys. Rev. D61 (2000) 124006 